\title{
Development of a method for the determination of the pressure balance piston fall rate
}

\author{
Lovorka Grgec Bermanec ${ }^{1}$, Davor Zvizdic ${ }^{1}$, Vedran Simunovic ${ }^{2}$ \\ ${ }^{1}$ Faculty of Mechanical Engineering and Naval Architecture, Laboratory for Process Measurement, 10000 Zagreb, Croatia \\ ${ }^{2}$ Faculty of Mechanical Engineering and Naval Architecture, Laboratory for Length Measurement, 10000 Zagreb, Croatia
}

\begin{abstract}
This paper describes a laboratory method for the determination of the pressure balance piston fall rate using a simple camera-based optical system with internally developed software. Measurements were carried out on three standard piston/cylinder units in the Croatian National Pressure Laboratory (LPM) using gas and oil as transmitting medium.

Measurement equipment, procedure and fall rate results for three sets of measurements are given, as well as an evaluation of the measurement uncertainty. Results were compared with other relevant measurements.
\end{abstract}

\begin{abstract}
Section: RESEARCH PAPER
Keywords: Pressure balance; Fall rate measurement; Sobel filter

Citation: Lovorka Grgec Bermanec, Marin Martinaga ${ }^{1}$, Davor Zvizdic, Vedran Simunovic, Development of method for determination of pressure balance piston fall rate, Acta IMEKO, vol. 3, no. 2, article 11, June 2014, identifier: IMEKO-ACTA-03 (2014)-02-11
\end{abstract}

Editor: Paolo Carbone, University of Perugia

Received April 15 $15^{\text {th }}, 2013$; In final form June 22 ${ }^{\text {nd }}, 2013$; Published June 2014

Copyright: @ 2014 IMEKO. This is an open-access article distributed under the terms of the Creative Commons Attribution 3.0 License, which permits unrestricted use, distribution, and reproduction in any medium, provided the original author and source are credited

Funding: (none reported)

Corresponding author: Lovorka G.Bermanec, e-mail: lovorka.grgec@fsb.hr

\section{INTRODUCTION}

The determination of the pressure balance piston fall rate is important for to several reasons. As an internal measure for quality assurance it indicates some deformation or changes in effective area [1], and in the "cross-float" calibration of other pressure balances where the fall rate is obtained when the two balances are connected and is compared with the natural fall rate. If the fall rates differ, small masses can be added to or subtracted from one of the pressure balance, and the measurements should be repeated until the fall rates agree. [2].

For periodical determination of the LPM standard unit fall rates and for internal quality assurance it was necessary to develop a simple, efficient, repeatable and precise enough method.

Since there is no standard procedure for this measurement, there was no limitation in selecting equipment. Piston rate of fall is usually determined with laser sensors or expensive optic. Further equipment that was also taken into consideration in this work comprises eddy current sensors and different cameras.

A simple camera has been chosen for analysing measurement possibilities regarding accuracy, accessibility and price.

\section{MEASUREMENT METHOD AND CALCULATION PROCEDURE}

Measurements were performed with an amateur camera (Nikon Digital Camera) equipped with appropriate lenses. A plane parallel gauge block with $1.5 \mathrm{~mm}$ thickness was used to relate relative motion to real displacement in millimetres. Before the measurement, while the piston was in stand-up position, a snap of the standard gauge block was taken.

Pictures were analysed using Matlab software which has inbuilt and predefined functions for various filters. In this measurement a Sobel filter was used. This filter is often used for edge detection. Edge detection enables to follow the relative movement of the pressure balance edges through continuous pictures.

After implementation of the Sobel filter, a simple method for transforming real thickness into pixel numbers was applied. From the number of pixels the movement between pictures can be calculated and converted into millimetres.

Two different results were obtained. For two consecutive measurements on the same gauge, the thickness of the standard gauge block was found to be 16 pixels and 15 pixels, 


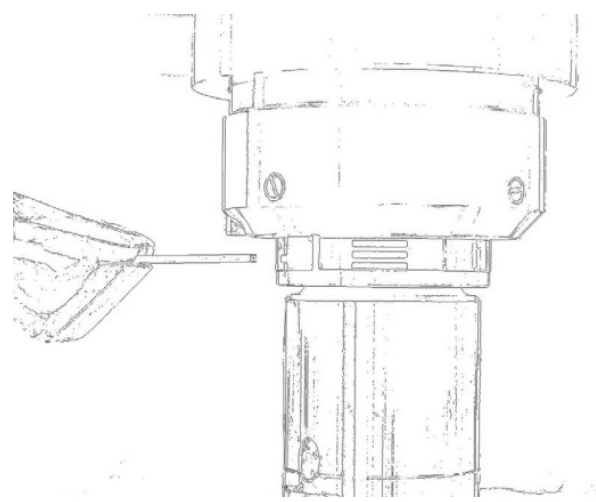

Figure 1. Computer image of the pressure balance and the plane parallel gauge block with applied Sobel filter.

respectively. This shows that a resolution of the described equipment of at least $0.1 \mathrm{~mm}$ was achieved.

The interval between two consecutive pictures was somewhat longer than one minute, so an adequate number of pictures can be acquired to achieve good accuracy. For the analysis of each measurement result, picture intervals of 3 seconds were used, which means 20 measurement points per minute.

The initial concept was to take 60 measurement points in a minute to precisely follow the movement of the pressure balance piston. Due to computer and camera limitations, the smallest possible interval between pictures with this equipment was 3 seconds. To avoid any contact with the camera, all adjusting parameters and the start of the photographing process were controlled by a computer connected to the camera.

Every setting was adjusted by appropriate software that allowed camera control via a cable. In this way a fixed position of the camera was assured, which is critical for the applied measurement method. After all the photographs were taken and have passed the Sobel filter (Figure 1), a series of 20 pictures for each measurement was obtained with information about the relative movement in pixels. To avoid accidental movement of the camera or imperfections of edges visible on the pictures, the $x$-axis was kept constant. In this way, possible distortions of the pictures are constant over the whole $y$-axis movement, avoiding errors. The relative motion in pixels on the $y$-axis was converted into $\mathrm{mm}$ for every two consecutive pictures.

Measurements were performed on three different effective areas of the piston/cylinder, including oil and gas pressure

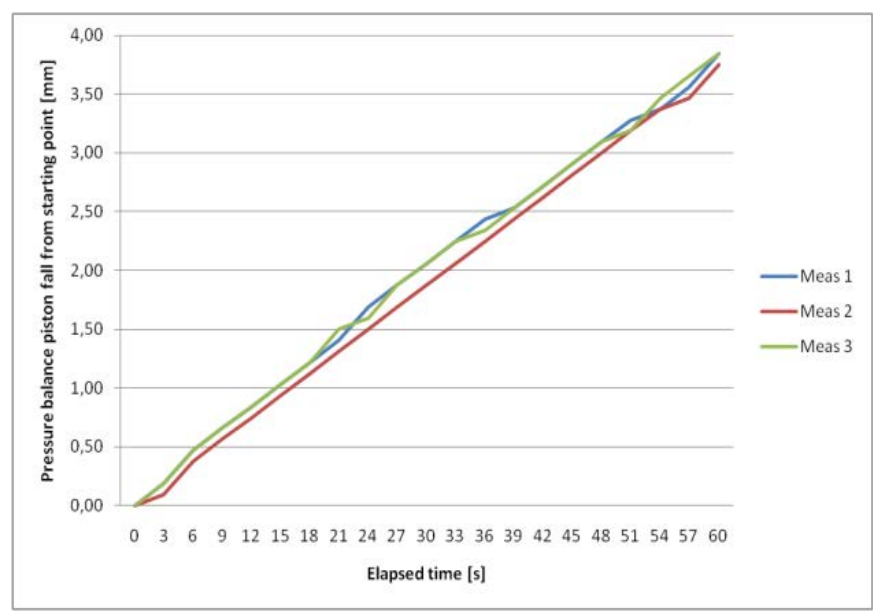

Figure 2. Determination of oil piston fall rate at 600 bar load.

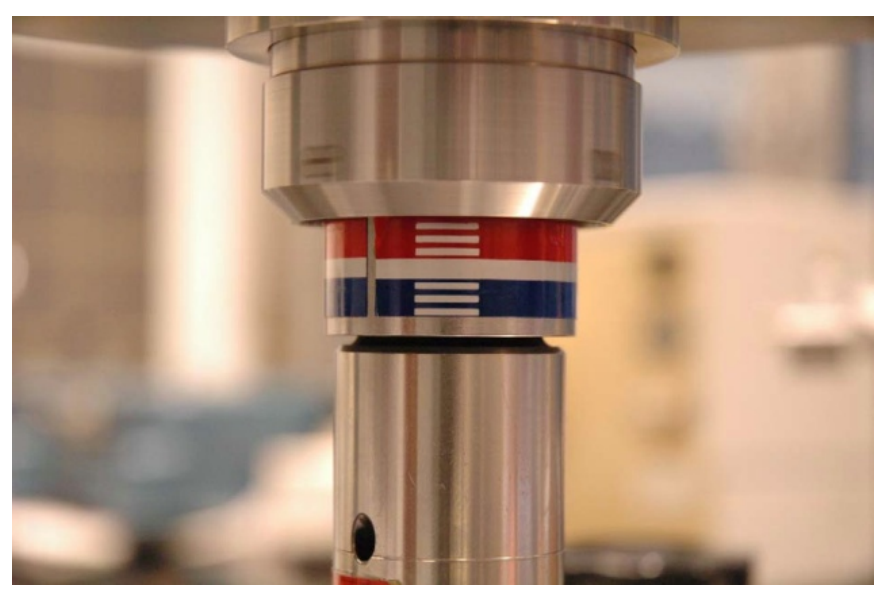

Figure 3. Start of measurement (first photograph) of the Budenberg pressure balance with 600 bar load.

balances (Figure 2). The oil pressure balance was designed by Budenberg with a double piston for 600 bar and 60 bar loads (Figure 3 and Figure 4). The gas pressure balance that was used in this work was a DHI PG7601 with 3 bar load. The DHI standard pressure balance is equipped with an internal fall rate sensor so we had an opportunity to compare results obtained between the proposed method and those obtained from the DHI pressure balance. The fall rate of the oil unit was compared with the last calibration certificates from the Physikalisch-Technische Bundesanstalt (PTB).

\section{FALL RATE RESULTS AND MEASUREMENT UNCERTAINTY EVALUATION}

In this paragraph the results for the three standard piston/cylinder units as well as the measurement uncertainty evaluation are presented.

The fall rate measurement uncertainty, $u_{\mathrm{F}}$, was evaluated as Type B uncertainty [3] taking into account the gauge block uncertainty, the camera resolution and the time measurements as the major influence quantities.

$$
u_{F}=\sqrt{u_{g}^{2}+u_{r}^{2}+u_{t}^{2}}
$$

where:

$u_{g} \quad$ - uncertainty of the plane parallel gauge block

$u_{r} \quad$ - uncertainty due to resolution

$u_{t} \quad$-uncertainty due to the time measurement

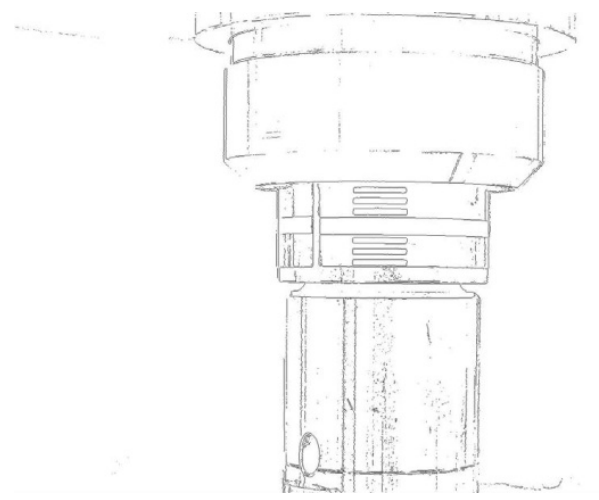

Figure 4. Start of the measurement (computer image) of the Budenberg pressure balance with 600 bar load after application of the Sobel filter. 
Table 1. Determination of oil piston fall rate at 600 bar load.

\begin{tabular}{|c|c|c|c|c|c|c|c|c|c|}
\hline \multirow[b]{2}{*}{$s$} & \multicolumn{3}{|c|}{ Measurement 1} & \multicolumn{3}{|c|}{ Measurement 2} & \multicolumn{3}{|c|}{ Measurement 3} \\
\hline & $\mathrm{x}$ & $y$ & $\underset{[\mathrm{mm}]}{\Delta y}$ & $\mathrm{x}$ & $y$ & $\underset{[\mathrm{mm}]}{\Delta y}$ & $x$ & $y$ & $\underset{[\mathrm{mm}]}{\Delta y}$ \\
\hline 0 & 760 & 352 & 0.00 & 760 & 349 & 0.00 & 760 & 353 & 0.00 \\
\hline 3 & 760 & 354 & 0.19 & 760 & 350 & 0.09 & 760 & 355 & 0.19 \\
\hline 6 & 760 & 357 & 0.47 & 760 & 353 & 0.38 & 760 & 358 & 0.47 \\
\hline 9 & 760 & 359 & 0.66 & 760 & 355 & 0.56 & 760 & 360 & 0.66 \\
\hline 12 & 760 & 361 & 0.84 & 760 & 357 & 0.75 & 760 & 362 & 0.84 \\
\hline 15 & 760 & 363 & 1.03 & 760 & 359 & 0.94 & 760 & 364 & 1.03 \\
\hline 18 & 760 & 365 & 1.22 & 760 & 361 & 1.13 & 760 & 366 & 1.22 \\
\hline 21 & 760 & 367 & 1.41 & 760 & 363 & 1.31 & 760 & 369 & 1.50 \\
\hline 24 & 760 & 370 & 1.69 & 760 & 365 & 1.50 & 760 & 370 & 1.59 \\
\hline 27 & 760 & 372 & 1.88 & 760 & 367 & 1.69 & 760 & 373 & 1.88 \\
\hline 30 & 760 & 374 & 2.06 & 760 & 369 & 1.88 & 760 & 375 & 2.06 \\
\hline 33 & 760 & 376 & 2.25 & 760 & 371 & 2.06 & 760 & 377 & 2.25 \\
\hline 36 & 760 & 378 & 2.44 & 760 & 373 & 2.25 & 760 & 378 & 2.34 \\
\hline 39 & 760 & 379 & 2.53 & 760 & 375 & 2.44 & 760 & 380 & 2.53 \\
\hline 42 & 760 & 381 & 2.72 & 760 & 377 & 2.63 & 760 & 382 & 2.72 \\
\hline 45 & 760 & 383 & 2.91 & 760 & 379 & 2.81 & 760 & 384 & 2.91 \\
\hline 48 & 760 & 385 & 3.09 & 760 & 381 & 3.00 & 760 & 386 & 3.09 \\
\hline 51 & 760 & 387 & 3.28 & 760 & 383 & 3.19 & 760 & 387 & 3.19 \\
\hline 54 & 760 & 388 & 3.38 & 760 & 385 & 3.38 & 760 & 390 & 3.47 \\
\hline 57 & 760 & 390 & 3.56 & 760 & 386 & 3.47 & 760 & 392 & 3.66 \\
\hline 60 & 760 & 393 & 3.84 & 760 & 389 & 3.75 & 760 & 394 & 3.84 \\
\hline
\end{tabular}

\subsection{Oil operated system up to $600 \mathrm{bar}$}

Measurements performed on the Budenberg standard pressure balance with 600 bar load has a high rate of fall, as expected. This was clearly visible without any equipment. Results can be compared with results given in the calibration certificate obtained from the PTB. These results were $(3.0 \pm$

Table 3. Determination of oil piston fall rate at 60 bar load.

\begin{tabular}{cccccccccc}
\hline & \multicolumn{3}{c}{ Measurement 1 } & \multicolumn{3}{c}{ Measurement 2 } & \multicolumn{2}{c}{ Measurement 3 } \\
\cline { 2 - 10 } & $\mathbf{x}$ & $\mathbf{y}$ & $\begin{array}{c}\mathbf{\Delta} \mathbf{y} \\
{[\mathbf{m m}]}\end{array}$ & $\mathbf{x}$ & $\mathbf{y}$ & $\begin{array}{c}\boldsymbol{\Delta} \mathbf{y} \\
{[\mathbf{m m}]}\end{array}$ & $\mathbf{x}$ & $\mathbf{y}$ & $\begin{array}{c}\boldsymbol{\Delta} \mathbf{y} \\
{[\mathbf{m m}]}\end{array}$ \\
\hline $\mathbf{0}$ & 760 & 492 & 0.0 & 760 & 487 & 0.0 & 760 & 487 & 0.0 \\
$\mathbf{3}$ & 760 & 492 & 0.0 & 760 & 488 & 0.1 & 760 & 487 & 0.0 \\
$\mathbf{6}$ & 760 & 492 & 0.0 & 760 & 488 & 0.1 & 760 & 487 & 0.0 \\
$\mathbf{9}$ & 760 & 492 & 0.0 & 760 & 488 & 0.1 & 760 & 487 & 0.0 \\
$\mathbf{1 2}$ & 760 & 492 & 0.0 & 760 & 488 & 0.1 & 760 & 487 & 0.0 \\
$\mathbf{1 5}$ & 760 & 493 & 0.1 & 760 & 488 & 0.1 & 760 & 487 & 0.0 \\
$\mathbf{1 8}$ & 760 & 493 & 0.1 & 760 & 488 & 0.1 & 760 & 488 & 0.1 \\
$\mathbf{2 1}$ & 760 & 493 & 0.1 & 760 & 488 & 0.1 & 760 & 488 & 0.1 \\
$\mathbf{2 4}$ & 760 & 493 & 0.1 & 760 & 488 & 0.1 & 760 & 488 & 0.1 \\
$\mathbf{2 7}$ & 760 & 493 & 0.1 & 760 & 488 & 0.1 & 760 & 488 & 0.1 \\
$\mathbf{3 0}$ & 760 & 493 & 0.1 & 760 & 489 & 0.2 & 760 & 488 & 0.1 \\
$\mathbf{3 3}$ & 760 & 493 & 0.1 & 760 & 489 & 0.2 & 760 & 488 & 0.1 \\
$\mathbf{3 6}$ & 760 & 494 & 0.2 & 760 & 489 & 0.2 & 760 & 488 & 0.1 \\
$\mathbf{3 9}$ & 760 & 494 & 0.2 & 760 & 489 & 0.2 & 760 & 488 & 0.1 \\
$\mathbf{4 2}$ & 760 & 494 & 0.2 & 760 & 489 & 0.2 & 760 & 489 & 0.2 \\
$\mathbf{4 5}$ & 760 & 494 & 0.2 & 760 & 489 & 0.2 & 760 & 489 & 0.2 \\
$\mathbf{4 8}$ & 760 & 494 & 0.2 & 760 & 489 & 0.2 & 760 & 489 & 0.2 \\
$\mathbf{5 1}$ & 760 & 494 & 0.2 & 760 & 489 & 0.2 & 760 & 489 & 0.2 \\
$\mathbf{5 4}$ & 760 & 494 & 0.2 & 760 & 489 & 0.2 & 760 & 489 & 0.2 \\
$\mathbf{5 7}$ & 760 & 494 & 0.2 & 760 & 490 & 0.3 & 760 & 489 & 0.2 \\
$\mathbf{6 0}$ & 760 & 495 & $\mathbf{0 . 3}$ & 760 & 490 & $\mathbf{0 . 3}$ & 760 & 490 & $\mathbf{0 . 3}$ \\
\hline & & & & & & & & &
\end{tabular}

Table 2. Fall rate uncertainty evaluation.

\begin{tabular}{|c|c|c|c|c|}
\hline $\begin{array}{l}\text { Influence } \\
\text { quantity }\end{array}$ & $\begin{array}{l}\text { Uncertainty } \\
\text { of the } \\
\text { influence } \\
\text { quantity }\end{array}$ & Factor & $\begin{array}{l}\text { Sensitivity } \\
\text { coefficient }\end{array}$ & $\begin{array}{l}\text { Contribution } \\
\text { to the } \\
\text { standard } \\
\text { uncertainty }\end{array}$ \\
\hline $\begin{array}{c}\text { Plane } \\
\text { parallel } \\
\text { gauge block }\end{array}$ & $0.1 \mu \mathrm{m}$ & $\sqrt{3}$ & 1 & $0.06 \mu \mathrm{m}$ \\
\hline Resolution & $0.1 \mathrm{~mm}$ & $\sqrt{3}$ & 1 & $57.8 \mu \mathrm{m}$ \\
\hline \multirow[t]{3}{*}{ Time } & $0.5 \mathrm{~s}$ & $\sqrt{3}$ & $0.06 \mathrm{~mm} / \mathrm{s}$ & $17.3 \mu \mathrm{m}$ \\
\hline & \multicolumn{2}{|c|}{ Fall rate uncertainty } & $u_{F}$ & $60 \mu \mathrm{m}$ \\
\hline & \multicolumn{2}{|c|}{$\begin{array}{l}\text { Expanded fall } \\
\text { rate measurement } \\
\text { uncertainty }(k=2)\end{array}$} & $U_{F}=2 \cdot u_{F}$ & $0.12 \mathrm{~mm}$ \\
\hline
\end{tabular}

0.5) $\mathrm{mm} / \mathrm{min}$. Results from the LPM first unit are given in Table 1.

From this result it can be seen that the fall rate is too large for a pressure balance classified in the accuracy class of 0.02 . The maximum piston fall rate defined in [1] is $1.5 \mathrm{~mm} / \mathrm{min}$.

The uncertainty estimation is given in Table 2, only for the first piston/cylinder unit, although it is calculated for each unit separately with different sensitivity coefficient for the time measurement.

\subsection{Oil operated system up to $60 \mathrm{bar}$}

The second measurement is performed on the same Budenberg oil unit but using a low pressure range up to 60 bar maximum load. Results from the PTB for this unit were $(0.26 \pm$ $0.10) \mathrm{mm} / \mathrm{min}$. Results obtained from the picture analysis are shown in Table 3.

Good agreement between the results of PTB and LPM can be observed.

Table 4. Determination of DHI PG7601 gas piston fall rate at 3 bar load.

\begin{tabular}{|c|c|c|c|c|c|c|c|c|c|}
\hline \multirow[b]{2}{*}{$s$} & \multicolumn{3}{|c|}{ Measurement 1} & \multicolumn{3}{|c|}{ Measurement 2} & \multicolumn{3}{|c|}{ Measurement 3} \\
\hline & $\mathrm{x}$ & $y$ & $\underset{[\mathrm{mm}]}{\Delta y}$ & $x$ & $y$ & $\underset{[\mathrm{mm}]}{\Delta y}$ & $x$ & $y$ & $\underset{[\mathrm{mm}]}{\Delta \mathbf{y}}$ \\
\hline 0 & 670 & 495 & 0.00 & 670 & 496 & 0.00 & 670 & 495 & 0.00 \\
\hline 3 & 670 & 495 & 0.00 & 670 & 496 & 0.00 & 670 & 496 & 0.09 \\
\hline 6 & 670 & 495 & 0.00 & 670 & 496 & 0.00 & 670 & 496 & 0.09 \\
\hline 9 & 670 & 496 & 0.09 & 670 & 496 & 0.00 & 670 & 496 & 0.09 \\
\hline 12 & 670 & 496 & 0.09 & 670 & 497 & 0.09 & 670 & 496 & 0.09 \\
\hline 15 & 670 & 496 & 0.09 & 670 & 497 & 0.09 & 670 & 497 & 0.19 \\
\hline 18 & 670 & 497 & 0.19 & 670 & 497 & 0.09 & 670 & 497 & 0.19 \\
\hline 21 & 670 & 497 & 0.19 & 670 & 497 & 0.09 & 670 & 497 & 0.19 \\
\hline 24 & 670 & 497 & 0.19 & 670 & 498 & 0.19 & 670 & 498 & 0.28 \\
\hline 27 & 670 & 498 & 0.28 & 670 & 498 & 0.19 & 670 & 498 & 0.28 \\
\hline 30 & 670 & 498 & 0.28 & 670 & 498 & 0.19 & 670 & 498 & 0.28 \\
\hline 33 & 670 & 498 & 0.28 & 670 & 499 & 0.28 & 670 & 499 & 0.38 \\
\hline 36 & 670 & 499 & 0.38 & 670 & 499 & 0.28 & 670 & 499 & 0.38 \\
\hline 39 & 670 & 499 & 0.38 & 670 & 499 & 0.28 & 670 & 499 & 0.38 \\
\hline 42 & 670 & 499 & 0.38 & 670 & 500 & 0.38 & 670 & 500 & 0.47 \\
\hline 45 & 670 & 500 & 0.47 & 670 & 500 & 0.38 & 670 & 500 & 0.47 \\
\hline 48 & 670 & 500 & 0.47 & 670 & 500 & 0.38 & 670 & 500 & 0.47 \\
\hline 51 & 670 & 500 & 0.47 & 670 & 501 & 0.47 & 670 & 500 & 0.47 \\
\hline 54 & 670 & 501 & 0.56 & 670 & 501 & 0.47 & 670 & 501 & 0.56 \\
\hline 57 & 670 & 501 & 0.56 & 670 & 501 & 0.47 & 670 & 501 & 0.56 \\
\hline 60 & 670 & 501 & 0.56 & 670 & 502 & 0.56 & 670 & 501 & 0.56 \\
\hline
\end{tabular}




\subsection{Gas operated standard system up to 3 bar}

Third set of measurements was performed on the gas operated DHI pressure balance with a maximum load of 3 bar. This unit is equipped with an internal fall rate sensor and all the results were directly compared. Results obtained after pictures analyses are shown in Table 4.

In this measurement, the relative movement was $0.094 \mathrm{~mm}$ for one pixel, and the internal fall rate sensor has a precision of $0.1 \mathrm{~mm}$. This prevented direct comparison. As it can be seen from the results in Table 3, the pressure balance fall rate is 0.56 $\mathrm{mm} / \mathrm{min}$, and the internal fall rate sensor started changing its value from $0.5 \mathrm{~mm}$ to $0.6 \mathrm{~mm}$ after one minute and three seconds.

The maximum piston fall rate for gas operated systems according to the OIML document is $1 \mathrm{~mm} / \mathrm{min}$.

Comparing the results in all three cases with results from the calibration certificates, as well as from comparison with the internal fall rate sensor in the DHI pressure balance, it can be concluded that new method is sufficiently accurate for further development.

\section{CONCLUSIONS}

An internal laboratory method for the determination of the fall rate was developed in the LPM, with a target uncertainty of $0.1 \mathrm{~mm} / \mathrm{min}$, using a camera based optical system.

The advantages of the proposed method are a simple and cheap measurement equipment.

Measurement results obtained with the proposed method show good agreement with other relevant measurements.

Disadvantages are found in the choice of lenses. Further development of the method focuses on the automation of the measurements.

\section{REFERENCES}

[1] 1994 OIML regulation R110, edition 1994(E) Pressure Balances (Paris: Organisation International de M'etrologie L'egale)

[2] Dadson R.S., Lewis S.L., Peggs G.N., The pressure balance: Theory and Practice, Ed.1., HMSO, London, 1982.

[3] ISO Guide to the Expression of Uncertainty in Measurement, Geneva: ISO, 1995 